\title{
Mycobacterium tuberculosis tlyA E59Q is Stable Compared to the Native Protein
}

\author{
V. B. Shivaleela ${ }^{1}$, ShaikKalimulla Niazi ${ }^{2}$, P. Sharada ${ }^{1 *}$ and K. Giri Gowda ${ }^{3 *}$ \\ ${ }^{1}$ Department of Biotechnology, Basaveshwara Engineering College, \\ Bagalkot-587102, Karnataka, India \\ ${ }^{2}$ Department of Biotechnology, Preparatory Health Sciences, Riyadh Elm University, \\ Riyadh, Saudi Arabia \\ ${ }^{3}$ Sampoorna International Institute of Agri.Science \& Horticulture Technology, \\ Mandya-571433, Karnataka, India
}

*Corresponding author

\section{A B S T R A C T}

Keywords

tlyA,

Mycobacterium

tuberculosis,

methyltransferase,

antibiotic

resistance,

mutation.

\section{Article Info}

Accepted:

22 August 2019

Available Online:

10 September 2019
The causative agent of human tuberculosis disease, mycobacterium tuberculosis (Mtb), infects one-third of the world population. Mycobacterium tuberculosis tlyA is an important protein and the mutation can cause resistance to antibiotics. Understanding the structure-function of mycobacterium tuberculosis tlyA is essential for preventing antibiotic resistance. Structure of Mycobacterium tuberculosis CTD of tlyA structure was solved, whereas its biological functions are not fully understood. Here, we studied tlyA protein by mutational analysis and molecular modelling studies. Further, purified tlyA is found to be very stable compared to the native tly $\mathrm{A}$ and retained its three dimensional structure. In the absence of full length tlyA structure, molecular model was generated by I-Tasser server using tlyA CTD crystal structure as a template. Generated model showed $90 \%$ of residues in the allowed region, $7 \%$ in additional allowed region and 3\% residues in disallowed region. These results reveal Mtb-tlyA NTD is important for its structure and function and E59Q is a critical residue for retaining the $\mathrm{N}$-terminus.

\section{Introduction}

Mycobacterium tuberculosis (Mtb) is one of the most infectious pathogens and causes about 1.3 million deaths and 10 million cases of new infections every year (WHO report, 2018).Drug resistant $M t b$ becoming more prevalent, major concern is the rising number of TB patients involving $M t b$ strains significantly increased are multidrug-resistant 
including resistant to isoniazid and rifampicin (Witek et al., 2017).

Understanding the modification in proteins for drug resistance is critical for the treatment of TB. Ribosome is one of the most common target so antibiotics in the cell and mutations in different components of ribosome are responsible for drug resistance. Several mutations in 23S and 16SrRNA genes, rRNA methyltransferases, and ribosomal proteins in different mycobacterial strains have been shown to be responsible for resistance to different drugs (Kumar et al., 2011). rRNA methyltransferases are strong candidates for drug targeting against mycobacteria. Resistance to antibiotics in $M t b$ can aquirevia mutation of tlyA, the gene encoding the protein tlyA (Rv1694), a proposed virulence factor. tly A belongs to a unique group of methyltransferases for which the loss of function confers bacterial antibiotic resistance. Many bacterial genera lack tlyA, the potent antibiotic activity of capreomycin is specific against Mtb. Capreomycin targets the ribosome of $M t b$ at the interface of the small and large subunits and requires ribosomal RNA (rRNA) methylation for optimal binding and thus inhibition of ribosome function (Kumar et al., 2011).

However, treatment of TB has become problematic not only because of the side effects of aminoglycosides but also because of the increased incidence of virulent, capreomycin-resistant $M t b$ strains generated by inactivation of tlyA (Kumar et al., 2011, Maus et al., 2005). Despite the critical role of tly $\mathrm{A}$ in capreomycin sensitivity, identification of inactivating mutations that cause resistance is lacking and our current understanding of tlyA structure and mechanism of action remains limited.

Despite the importance of tlyA activity in capreomycin action and resistance, many molecular details of the tlyA mechanism of action remain largely unknown. Therefore, detailed molecular studies of tlyA are urgently required for the better understanding of the resistance. Previous studies demonstrated that Mtb-tlyA folds into two stable structural domains connected by a protease-sensitive linker, with rRNA binding and SAM binding/methyltransferase activities expected to reside in the amino- and carboxyl terminal domains (NTD and CTD), respectively. Studies also showed the role of a minimalist $\mathrm{N}$-terminal domain in recruiting Rv2966c to ribosome to carry out its methyltransferase function.

Hence, a comprehensive understanding of tlyA full length protein structure and its mutational studies is required for understanding the resistance in $M t b-t l y A$. In the present study, we mutated critical residue E59Q of tlyA and the intact protein containing NTD and CTD was purified. Molecular model Mtb-tlyA was generated using (PDB ID:5EOV) as template for clear understanding the structure-function relationship.

\section{Materials and Methods}

\section{Bacterial strains and plasmid}

pETDuet-1 vector system was obtained from Invitrogen (California, USA) and was used according to the manufacturer's instructions. E.coli DH5 $\alpha$ and E.coliBL-21competent cells were obtained from Invitrogen (California, USA).

\section{PCR amplification of tlyAnativeandE59Q}

Oligonucleotide primers used for amplification of $M t b$-tlyA native were designed based on the tly $\mathrm{A}$ sequence of Mycobacterium tuberculosis strain $\mathrm{H} 37 \mathrm{Rv}$ deposited in the genome database (NCBI accession no.AQO55200.1). The sequence of 
the forward primer was 5'-GCGGAATT CGATGGCACGACGTGCCCGCGTT-3' and the reverse primer was 5'-TATGGTAC CTTACGGGCCCTCGCTAATCGCACG-3'.

The reaction mixture used for gene amplification contained $2 \mu \mathrm{l}$ of $5 \mathrm{X}$ phusion buffer supplied with the enzyme, $200 \mu \mathrm{M}$ of each dNTPs, $0.5 \mu \mathrm{M}$ of each primer, $150 \mathrm{ng}$ of DNA template, and of $0.02 \mathrm{U} / \mu \mathrm{l}$ phusion DNA polymerase (New England Biolabs, Massachusetts, USA) and water to a final volume of $20 \mu \mathrm{l}$. After optimizing the conditions for polymerase chain reaction (PCR), the gene was successfully amplified using the following PCR conditions: $98{ }^{\circ} \mathrm{C}$ for $30 \mathrm{sec}$ followed by 30 cycles of denaturation at $98{ }^{\circ} \mathrm{C}$ for $10 \mathrm{sec}$, annealing at $60{ }^{\circ} \mathrm{C}$ for 30sec, extension at $72{ }^{\circ} \mathrm{C}$ for $30 \mathrm{sec}$ and the final extension was carried out at $72{ }^{\circ} \mathrm{C}$ for 10 min on a PTC-100 Thermocycler (M.J. Research, Watertown, MA). The PCR product was analysed by electrophoresis on a $1 \%$ agarose gel and showed a DNA band of the expected size which was purified using a gel extraction kit (Thermofisher Scientific, Waltham, Massachusetts, USA).Mutant of tlyAE59Q was designed,the sequence of the forward primer was 5'-ACCGTGGTGA CCGACAGTCAACGCGCCTGGGTATCGC GC-3'and the reverse primer was 5'GCGCGATACCCAGGCGCGTTGACTGTC GGTCACCACGGT-3.Sequential two step PCR amplification was performed for the tlyAE59Q mutant using forward and reverse primers of the native tlyA.

\section{Cloning and DNA sequencing}

Purified PCR product was cloned into plasmid DNA using the pETDuet-1 vector system (Novagen, Wisconsin, USA). The PCR product and pETDuet-1 vector was digested with restriction enzymes EcoRI and KpnI for $2 \mathrm{~h}$ at $37^{\circ} \mathrm{C}$, product was purified. $2 \mu \mathrm{l}$ of purified PCR product was mixed with $0.5 \mu \mathrm{l}$ linearized pET-Duet-1 cloning vector in presence of $0.5 \mu \mathrm{l}$ T4 DNA ligase (Thermofisher Scientific, Massachusetts, USA) and incubated overnight at $16{ }^{\circ} \mathrm{C}$. Then the ligation mixture was directly used for the transformation of $\mathrm{CaCl}_{2}$ competent DH5acells by heat shock method (Inoue et al., 1990) Transformed cells were screened by ampicillin resistant, blue-white selection with $\mathrm{X}$-gal and IPTG. Colony PCR was performed to screen positive colonies. Positive colonies were picked, grown overnight in $5 \mathrm{ml}$ of LB broth at $37{ }^{\circ} \mathrm{C}$ and plasmids were isolated using commercial mini-prep kit (GCC Biotech, Joychandipur, West Bengal, India). Restriction digestion screening of the isolated plasmids were done to select the construct containing the correct size insert and selected constructs were sequenced. Sequencing was performed using vector specificT7 promoter primer.

\section{Expression and purification}

The pETDuet-1 plasmid containing E59Q mutant gene was transformed into $E$. coli BL21 (Star) competent cells. For protein expression, transformed BL21 (Star) cells were grown at $37^{\circ} \mathrm{C}$ to an optical density of 0.6 at $600 \mathrm{~nm}$ (OD600) and induced with 0.5 $\mathrm{mM}$ isopropyl- $\beta$-thiogalactopyranoside (IPTG). Induced cultures were transferred to $18^{\circ} \mathrm{C}$ and cells were grown for 12-14 h. Cells were harvested by centrifugation at 18,000 rpm at $4^{\circ} \mathrm{C}$ and cell pellets were stored at $20^{\circ} \mathrm{C}$ until further use. For protein purification, cell pellets from 1 litre culture were resuspended in $20 \mathrm{ml}$ of ice cold binding buffer containing 50 mMTrisHCl $(\mathrm{pH} 7.5)$, $300 \mathrm{mM}$ sodium chloride, $10 \%$ glycerol (v/v) and $5 \mathrm{mM} \beta$-mercaptoethanol. Lysozyme was added to a final concentration of $100 \mathrm{mg} / \mathrm{ml}$ and kept on rocking platform at $4^{\circ} \mathrm{C}$ for 45 min. PMSF was added immediately after the lysis $(0.2 \mathrm{mM})$. Cells were disrupted by sonication on ice with $50 \%$ amplitude and a pulse of $20 \mathrm{sec}$ on and $60 \mathrm{sec}$ off for $15 \mathrm{~min}$. 
The lysate was centrifuged at 18,000 rpm for $1 \mathrm{~h}$ at $4^{\circ} \mathrm{C}$ to separate supernatant from cell debris. The supernatant was loaded onto $5 \mathrm{ml}$ HisTrap HP affinity column pre-equilibrated with the binding buffer. Protein was eluted by running a linear gradient of $0-1000 \mathrm{mM}$ imidazole in $60 \mathrm{ml}$ of buffer A [50 mMTris $\mathrm{HCl}$ (pH 7.5), $1 \mathrm{M}$ imidazole, $300 \mathrm{mM}$ sodium chloride and $10 \%$ glycerol $(\mathrm{v} / \mathrm{v})]$ at a flow rate of $1 \mathrm{ml} / \mathrm{min}$. Eluted fractions were analyzed on sodium dodecyl sulfate-polyacrylamide gel electrophoresis (SDS-PAGE) and fractions containing pure protein were pooled together. Fractions containing E59QtlyAwas concentrated using a $10 \mathrm{kDa}$ cut-off Amicon Ultra-15 concentrator (Millipore, Bedford, Massachusetts, USA).

\section{Gel filtration Chromatography}

The concentrated protein was loaded onto HiLoad 16/60 prep grade Superdex75 sizeexclusion chromatography column preequilibrated with buffer containing $20 \mathrm{mMTris}-\mathrm{HCl}$ (pH 7.5), $1 \mathrm{M} \mathrm{NaCl}, 10 \%$ (v/v) glycerol and $5 \mathrm{mM} \beta$-mercaptoethanol using AKTA purification system (GE Healthcare). Protein was allowed to pass through the column at a rate of $0.8 \mathrm{ml} / \mathrm{min}$. The major peak fractions containing pure protein were pooled and concentrated. Homogeneity of purified E59QmutantlyA protein was analysed on $15 \%$ SDS-PAGE. Protein concentration and yield were determined using the Bio-Rad protein assay kit with bovine serum albumin (BSA) as a standard.

\section{SDS-PAGE}

SDS-PAGE was performed using $15 \%$ acrylamide gels, followed by the method of Laemmli (1970). The expressed soluble fractions were diluted with the sample buffer and boiled for 3 min before loading. A Gene Ruler $1 \mathrm{~kb}$ protein marker was used as a broad range protein standard to estimate the molecular weight of the proteins (Thermofisher Scientific, Waltham, Massachusetts, USA). The protein sample was separated at room temperature with a current of $20 \mathrm{~mA}$. The proteins were stained with Coomassie brilliant blue G-250 (Bio-Rad, Hercules, California, United States).

\section{Western blot analysis}

Western blot analysis was performed with the purified proteins separated by SDS-PAGE were transferred onto a polyvinylidene fluoride membrane using a mini trans-blot electrophoretic transfer cell (Bio-Rad, Hercules, California, United States). To detect the His-tagged recombinant protein, a Hisprobe (H-3) monoclonal anti-body (Santa Cruz) and a goat anti-mouse lgG-HRP (Santa Cruz) were used as the primary and secondary antibodies, respectively.

\section{Circular Dichroism studies}

Measurements of tlyAE59Q mutant was performed using a Chirascan CD spectrometer (Applied Photophysics). Cuvette path length used was $1 \mathrm{~mm}$, and sample concentrations was $0.80 \mathrm{mg} / \mathrm{ml}$. Protein buffer contained 10 $\mathrm{mM}$ sodium phosphate, $\mathrm{pH} 8.0,100 \mathrm{mMNaCl}$. The purity of samples were checked by SDSPAGE and size-exclusion chromatography. Each spectrum was averaged from four repeated scans ranging between 190 and 260 $\mathrm{nm}$ at a scan rate of $1.25 \mathrm{~nm} / \mathrm{s}$. Raw data were corrected by subtracting the contribution of the buffer to the signal.

\section{tlyA sequence analysis}

The sequence of Mycobacterium tuberculosis tlyA, along with other tlyAs were retrieved from NCBI database (Pruitt et al., (2005)). Sequence identity was verified by doing homology searches using the basic local alignment search tool (BLAST) algorithm 
(Stephan et al., 1990). Primary structure analysis of tlyA was done using the ProtP aramtool.

(http://www.expasy.ch/tools /protparam.html). The Clustal Omega multiple sequence alignment program was used to align the tlyA sequences (Sievers et al., 2011). The ESPript server was used for generating secondary structure elements and to produce a representation of the sequence alignment (Gouetet al., 1999).

\section{Comparative molecular modelling of $t$ ty $\mathrm{A}$}

Homology modeling for tlyA-Mtb was performed in the following sequential steps: template selection from Protein Data Bank (PDB), sequence-template alignment, model building, model refinement and validation. Template search for Mtb-tlyA was done using NCBI-BLAST search tool against PDB database. PDB format files of the crystal structure of CTD of $M t b$-tlyA which is available in the protein data bank (PDB ID: 5EOV) was downloaded. Mtb-tlyA CTD crystal structure was used as a template to generate a comparative 3D model of Mtb-tlyA full length by I-Tasser Server (Roy et al., 2010). I-Tasser Server generated several preliminary models which were ranked based on their C-scores. Five sets of models having lowest C-scores were selected and stereochemical quality of each was assessed by PROCHECK (Laskowski et al.,1993). The model with the least number of residues in the disallowed region was further refined for relieving steric clashes and improper contacts. Loop refinement tool of MODELLER was used in an iterative fashion to refine the loop conformation of the model. Structural validation after each loop refinement step was done using ERRAT plot which gives a measure of the structural error at each residue in the protein. This process was repeated iteratively until most of the amino acid residues were below 95\% cut-off value in ERRAT plot (Colovos andYeates, 1993). The refined model was further validated by VERIFY-3D of SAVES server (http://nihserver.mbi.ucla.edu/SAVES/).

ProSA 2003 was used to evaluate the generated 3D structure model of protein for potential errors (Wiederstein and Sippl, 2007).Model for visualization and superimposition of the model with the crystal structure of tlyA CTD was generated using PyMOL (DeLano 2002).

\section{Results and Discussion}

\section{Cloning and primary structure analysis}

The gene search of Mycobacterium Genome Database Sequences for tlyA reveals a single copy of gene in Mycobacterium species. To clone E59Qmutant from $M t b$, oligonucleotide primers were designed based on the gene sequence of tlyA. PCR was carried out and a 204bpfragment was amplified from $M t b$ H37Rv chromosomal DNA. The PCR product was purified by gel extraction method, cloned into pETDuet-1vector and sequenced. The clone thus obtained was confirmed by DNA sequencing. Such a cloning strategy resulted in the expression ofMtb-tlyAE59Qwith 16 additional amino acids (MGSSHHHHH HSQDPNS) generated when expressed in E.coli.

BLASTP search showed that the primary structure of Mtb-tlyA has highest sequence identity with mycobacterium canettitlyA, which showed 99\% sequence identity, whereas other mycobacterium species showed sequence identity of over $80 \%$. Sequence alignment of different tlyAproteins were represented in Figure. 1.

Nucleotide sequence analysis of cloned $M t b$ tlyA encodes for 268 amino acids with a predicted molecular mass of $\sim 29.9 \mathrm{kDa}$ including 16 additional amino acids, molecular weight of E59QtlyA is consistent 
with the tlyA native protein published (Rahman et al., 2010).

\section{Expression and purification}

To achieve high level expression of $M t b-$ tlyAE59Q,pETDuet-1 vector system was used as the expression vector which harbours a strong promoter, T7. pETDuet-1-Mtb-tlyA E59Q, with 16 additional amino acids including six histidines was transformed to
E.coli strains BL21 (star). The recombinant protein expression level was high when overproduced. Soluble form of the protein was detected in the BL21 (star) strain, His-tagged tlyAE59Q was confirmed by Western blot analysis using $\alpha$-his antibody (Figure.2B). To identify optimum temperature for protein production, over expression of $M t b$-tlyA from (BL-21(star) was analyzed with different temperature ranging from $16-37^{\circ} \mathrm{C}$ after $12 \mathrm{~h}$ of induction with IPTG (Data not shown).

Fig.1 Multiple sequence alignment oftlyA from different organisms was generated using Clustal Omega and figure was prepared using ESPript 3.0.
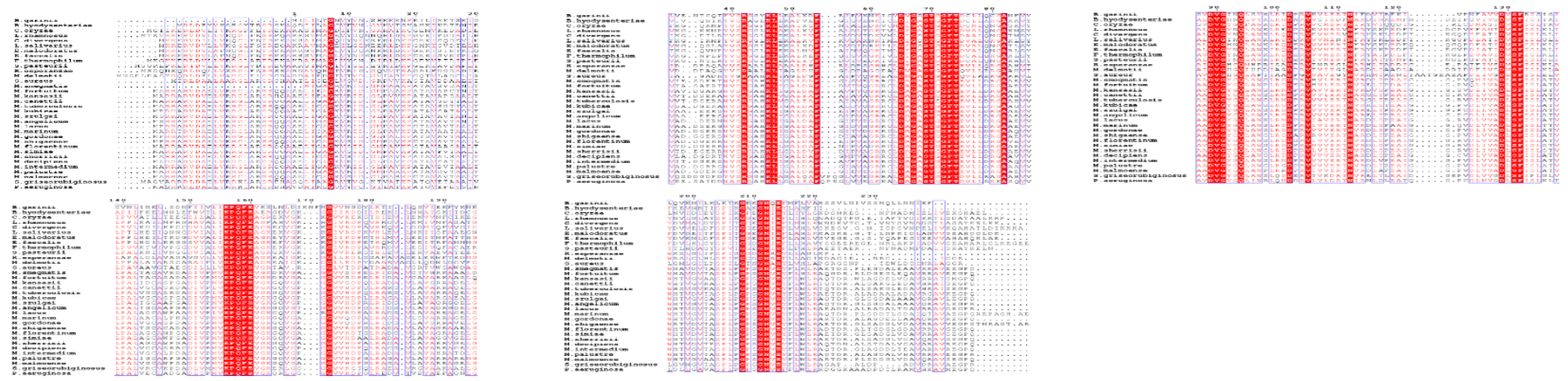

Fig.2 (A) Purification profile of Mtb-tlyA E59Q. (B) Western blot identification of His-Mtb-tlyA E59Q by $\alpha$-his antibody.

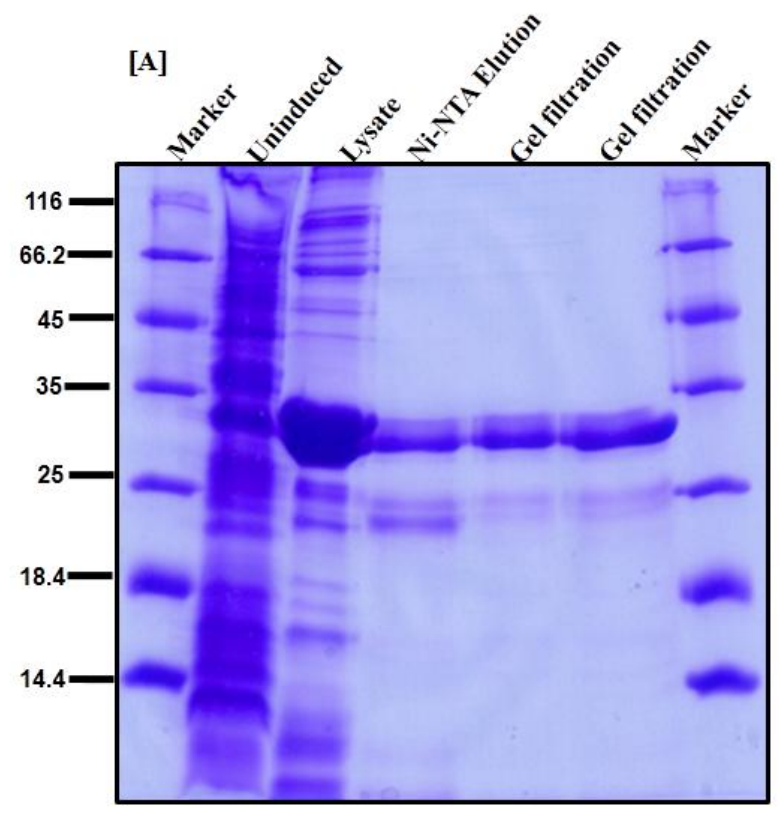

[B] 
Fig.3 CD spectroscopic analysis $M t b$-tlyAE59Q. CD studies of $M t b$-tlyA shows $\alpha / \beta$ pattern of structured protein.

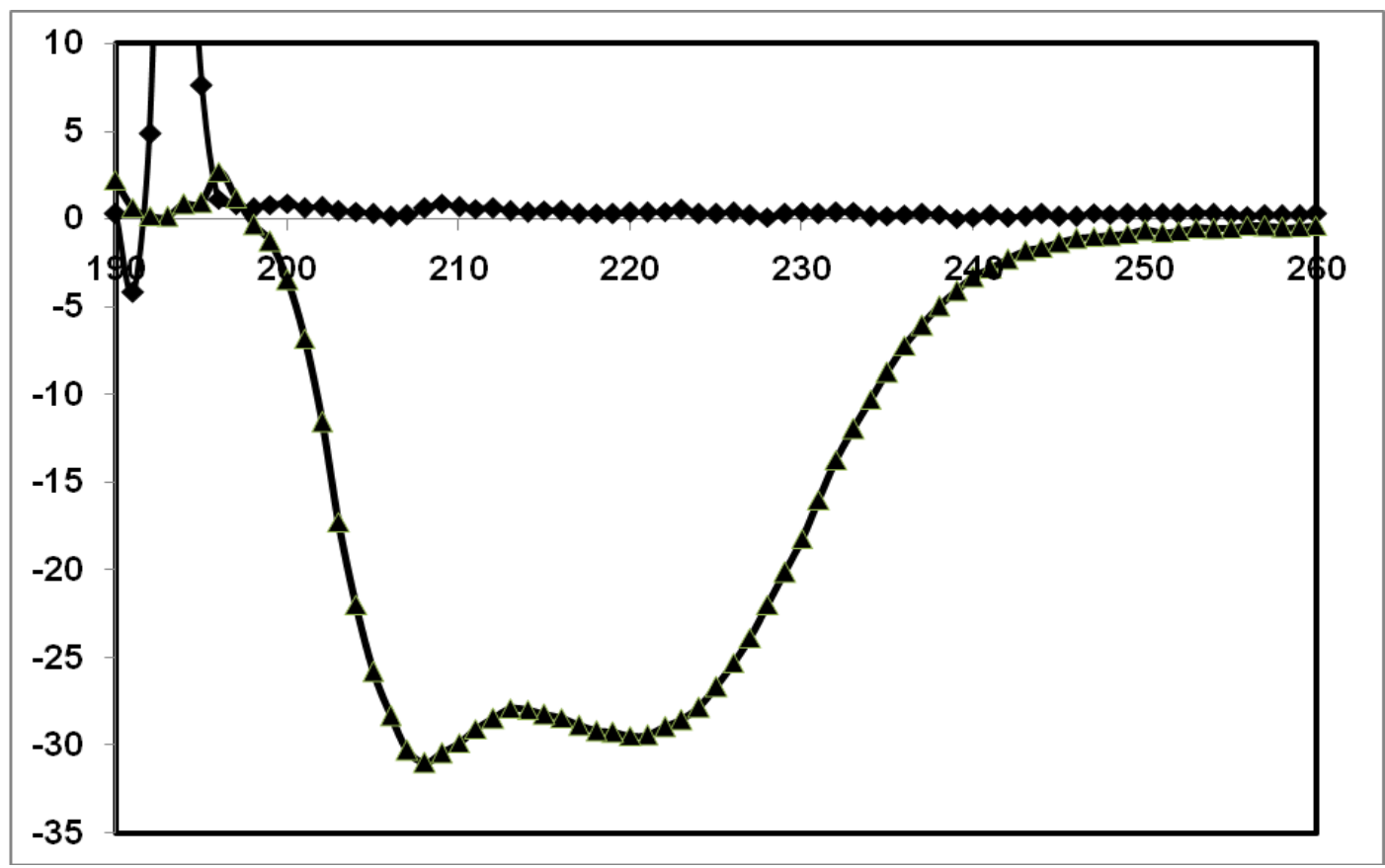

Fig.4 Mass spectrum of Mtb-tlyA E59Q obtained by MALDI-TOF.

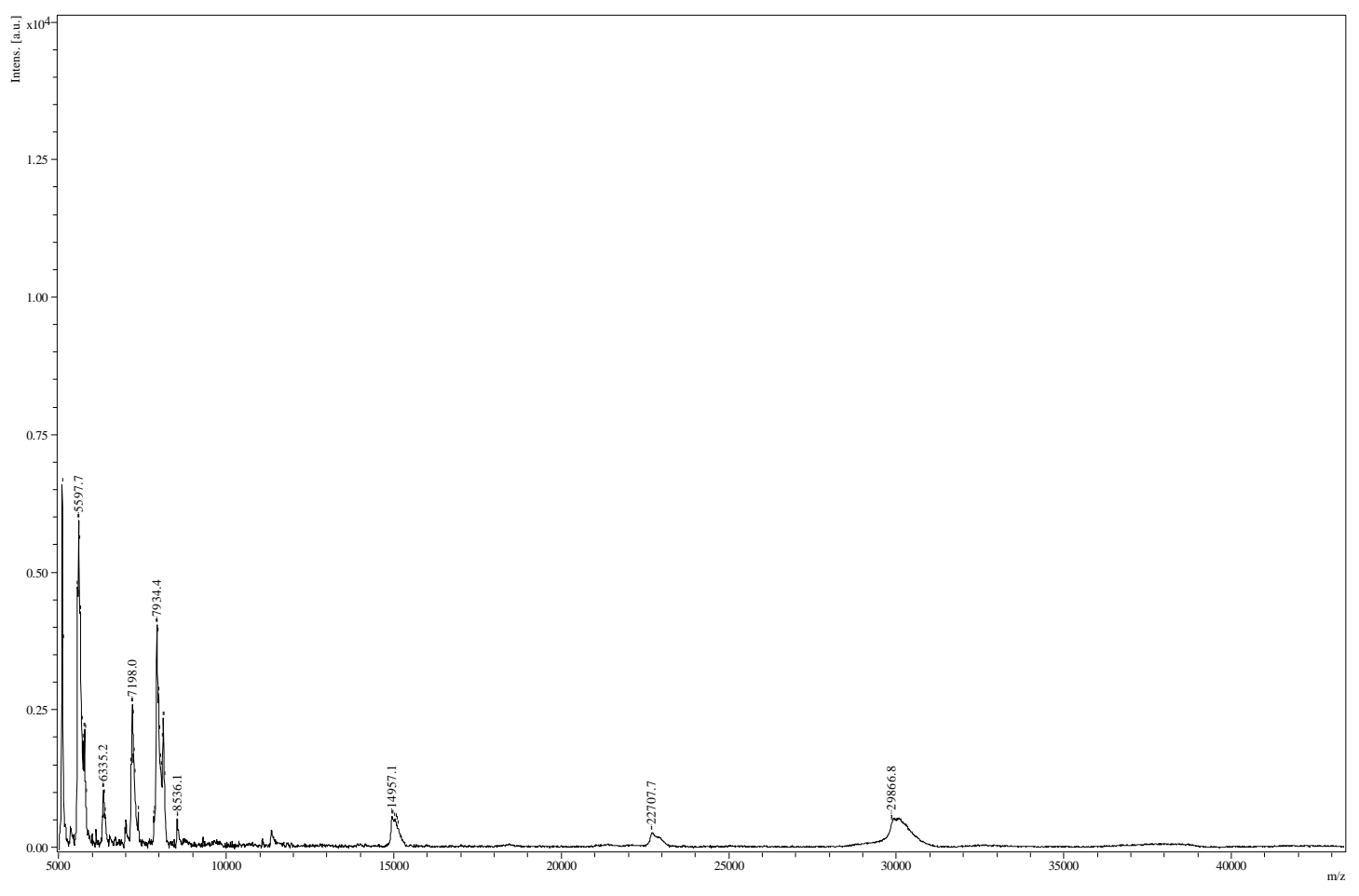


Fig.5 (A) $M t b$-tlyA generated model by I-Tasser Server (B) Superposition of $M t b$-TlyA model over crystal structure of $M t b$-TlyA CTD.

A.

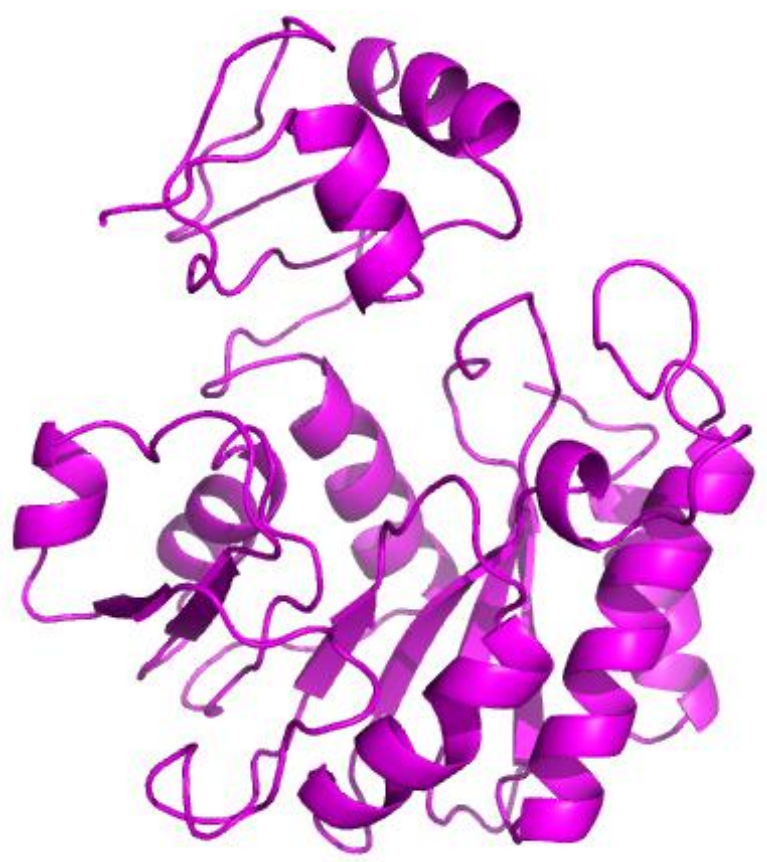

His-tlyAE59Q at the N-terminus which facilitated purification by $\mathrm{Ni}-\mathrm{NTA}$ affinity chromatography. Gel filtration chromatography was performed after $\mathrm{Ni}^{2+}$ affinity chromatography, where we could purifytlyAE59Q to homogeneity. The native protein reported to have autocatalytic activity (Witek et al., 2017), whereas E59Q did not show any autocatalytic activity. Gel filtration profile showed single predominant peak indicating the Mtb-tlyAE59Qis homogenous and which was further confirmed by SDSPAGE (Figure.2A). Fractions corresponding to protein on SDS-PAGE were pooled, concentrated and stored in $-80^{\circ} \mathrm{C}$.

The CD spectrum of His-tlyA native and E59Q showed the pattern of well folded protein with a mixed $\alpha / \beta$ structure and protein was quite stable even after storage in room temperature (Figure.3) (Whitmore et al.,
B.

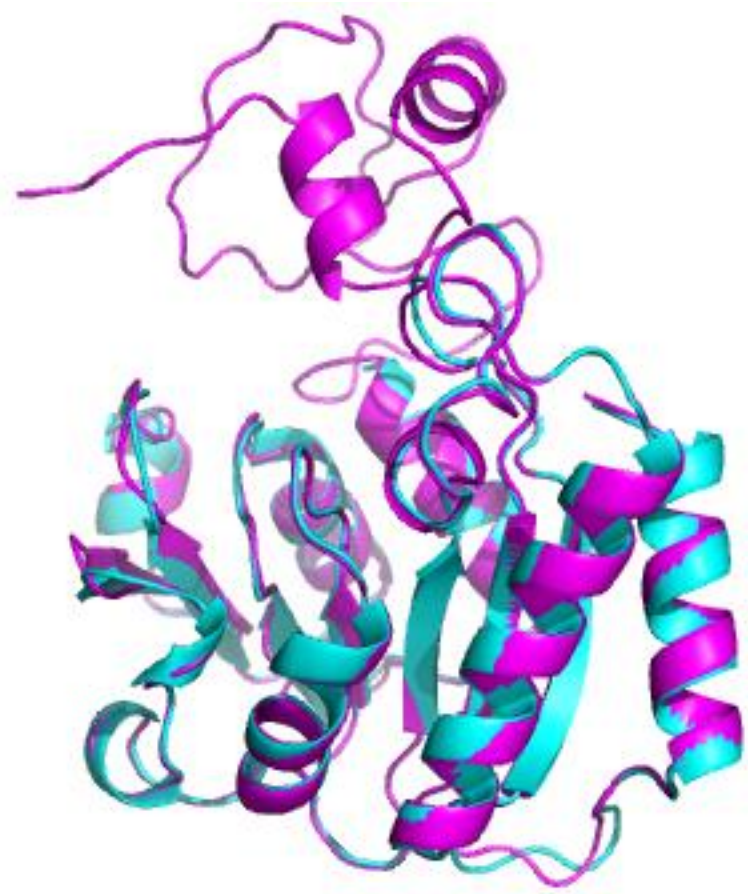

2008). Mass spectrometry analysis indicated the protein is $\sim 30 \mathrm{kDa}$ which is evident from the previous reports (Witek et al., 2017) (Figure.4).Previous studies indicated that Glu $^{59}$ surface exposed amino acid separates between NTD and CTD of Mtb-tlyA. Studies indicated that NTD and CTD were intact even after cleavage under the solution conditions (Witek et al., 2017).

\section{Molecular Model of tlyA full length protein}

Previous structural studies of $M t b$-tlyA CTD showed RrmJ/FtsJ Rossman-like methyltransferase fold and also overlays well with other Class I methyltransferases (Witek et al., 2017).

Studies also indicated that methyltransferase fold of tlyA is not sufficient for SAM binding, and thus, one or more amino acids of the 
aminoterminal residues of tlyA play an important role in SAM binding (Witek et al., 2017). Since Mtb-tlyA NTD plays an important role and is dispensable for interaction with the substrate, Mtb-tlyA Nterminus structure plays an important role.

Mtb-tlyA full length model was generated using crystallographic 3D structure of $M t b-$ tlyA CTD (PDB ID:5EOV) as template (Figure.5). The generated model was subjected to refinement, loop modeling and energy minimization. PROCHECK, Verify$3 \mathrm{D}$ and ERRAT plot were used for determining the stereo-chemical parameters of the energy minimized model of Mtb-tlyA.

Ramachandran plot of the 3D model generated by PROCHECK shows $90 \%$ in allowed region, $7 \%$ in generously allowed region and $3 \%$ in disallowed region. ERRAT plot gives an overall quality factor of 94.966 to the modeled structure. ProSA(2003) analysis showed that protein folding energy of our modeled structure is in good agreement with that of the template.

\section{Acknowledgement}

One of the author Shivaleela acknowledge TEQIP-III to Basavehwar Engineering College (A), Bagalkot for the financial support.

\section{References}

Colovos C andYeates TO (1993) Verification of protein structures:patterns of nonbonded atomic interactions. Protein Sci. 2:1511-1519.

DeLano WL (2002) ThePyMOL Molecular Graphics System. DeLano Scientific, San Carlos, CA, USA. http://www.pymol.org.

Gasteiger E, Gattiker A, Hoogland C, Ivanyi I, Appel RD and Bairoch A (2003)
ExPASy: the proteomics server for indepth protein knowledge and analysis. Nucl Acids Res 31:3784-3788.

Gouet P, Courcelle E, Stuart DI and Metoz F (1999) ESPript:analysis of multiple sequence alignments in PostScript. Bioinformatics 15:305-308.

Inoue H, Nojima H andOkayama H. (1990) High efficiency transformation of Escherichia coli with plasmids, 30: 96, 23-28.

Kumar A, Saigal K, Malhotra K, Sinha KM, and Taneja B (2011) Structural and Functional Characterization of Rv2966c Protein Reveals an RsmDlike Methyltransferase from Mycobacterium tuberculosis and the Role of Its N-terminal domain in target recognition. The Journal of Biological Chemistry, 286:22, 19652-19661.

Laemmli U (1970) Denaturing (SDS) discontinuous gel electrophoresis, Nature. 277:680-685.

Larkin MA, Blackshields G, Brown NP, Chenna R, McGettigan PA, McWilliam H, Valentin F, Wallace IM, Wilm A, Lopez R, Thompson JD, Gibson TJ and Higgins DG. (2007). Clustal W and Clustal X version 2.0. Bioinformatics, 23:2947-2948.

Laskowski RA, MacArthur MW, Moss DS and Thornton JM (1993) PROCHECK: a program to check the stereochemical quality of protein structures. J.ApplCrystallogr 26:283-291.

Maus CE, Plikaytis BB and Shinnick TM (2005) Mutation of tlyA confers capreomycin Resistance in mycobacterium tuberculosis. Antimicrobial agents and Chemotherapy, Feb. 2005:571-577.

Pruitt KD, Tatusova $\mathrm{T}$ and Maglott DR (2005). NCBI Reference Sequence (RefSeq): a curated non-redundant sequence database of genomes, 
transcripts and proteins. Nucleic Acids

Res. 33 (Database issue):501-4.

Rahman A, Srivastava SS, Sneh A, Ahmed N, and Krishnasastry MV (2010) Molecular characterization of tlyA gene product, Rv1694 of Mycobacterium tuberculosis: a nonconventional hemolysin and a ribosomal RNA methyl transferase. BMC Biochem. 11:35.

Roy A, Kucukural A, Zhang Y (2010) ITASSER: a unified platform for automated protein structure and function prediction. Nature Protocols, 5: 725-738.

Sievers F, Wilm A, Dineen DG, Gibson TJ, Karplus K, Li W, Lopez R, McWilliam H, Remmert M, Söding J, Thompson JD, Higgins DG (2011). Fast, scalable generation of high-quality protein multiple sequence alignments using Clustal Omega. Molecular Systems Biology 7:539.

Stephen A, Warren G, Webb M, Eugene M and David L(1990). Basic local alignment search tool. Journal of Molecular Biology.215(3): 403-410.

Whitmore L and Wallace BA (2008) Protein secondary structure analyses from circular dichroism spectroscopy: methods and reference databases. Biopolymers 89, 392-400.

Wiederstein M and Sippl MJ (2007) ProSAweb: interactive web service for the recognition of errors in threedimensional structures of proteins. Nucleic Acids Res 35:407-W410.

Witek MA, Kuiper EG, Minten E, Crispell EK and Conn GL (2017) A Novel Motif for S-Adenosyl-L-methionine binding by the ribosomal RNA methyltransferase tlyA from Mycobacterium tuberculosis. The Journal Of Biological Chemistry. 292:5, 1977-1987.

World Health Organization (2018) Global Tuberculosis Report 2018, World Health Organization, Geneva, Switzerland.

\section{How to cite this article:}

Shivaleela, V. B., ShaikKalimulla Niazi, P. Sharada and Giri Gowda, K. 2019. Mycobacterium tuberculosis tlyA E59Q is Stable Compared to the Native Protein. Int.J.Curr.Microbiol.App.Sci. 8(09): 2203-2212. doi: https://doi.org/10.20546/ijcmas.2019.809.254 